\title{
Aplicación de los ejes de Schwartz como metodología de prospectiva tecnológica al modelo universitario-empresa en el contexto colombiano
}

\author{
Mauricio Montoya Peláez \\ Politécnico Colombiano Jaime Isaza \\ Cadavid \\ mmontoya@elpoli.edu.co
}

\author{
Yenny Alejandra Aguirre Álvarez \\ Institución Universitaria Salazar y Herrera \\ yenny.aguirre@salazaryherrera.edu.co \\ Abdul Zuluaga Mazo \\ Politécnico Colombiano Jaime Isaza \\ Cadavid \\ azuluaga@elpoli.edu.co
}

\author{
Isabel Cristina Arango \\ Politécnico Colombiano Jaime Isaza \\ Cadavid \\ isabelarango@elpoli.edu.co
}

(Tipo de Artículo: Reflexión. Recibido el 19/12/2017. Aprobado el 21/02/2017)

Resumen. Han sido numerosos los trabajos generados desde la academia con el fin de evidenciar las relaciones existentes entre Empresa-Universidad-Gobierno. Este artículo pretende abordar la metodología de los Ejes de Schwartz como una de las estructuras más completas y acertadas que evidencia el modelo colaborativo existente entre estos tres actores. Para esta construcción se parte de una terminología clave, se determina el problema de investigación, luego se plantea el alcance con sus respectivos objetivos, después se realiza el desarrollo metodológico por medio de la aplicación de los ejes al contexto colombiano; para lo que se construye una lista del tema de estudio, definición del mapa con los ejes y futuros posibles, luego se analizan los resultados, y finalmente se generan conclusiones y recomendaciones. Como resultado principal de esta reflexión, se evidencia una fuerte tendencia en la transformación hacia una universidad empresarial, haciendo hincapié en la formación y la toma de riesgos, donde algunas empresas actúan incluso como universidades.

Palabras clave. Ejes de Schwartz, Empresa, Gobierno, Prospectiva tecnológica, Triple hélice, Universidad.

\section{Application Schwartz Shafts as the methodology for prospective technological University-Enterprise model in the context colombian}

\begin{abstract}
Many studies have been generated from the academy in order to demonstrate the relationship between Company-UniversityGovernment. This article aims to address the Axes methodology Schwartz as one of the most complete and accurate evidence the existing structures collaborative model between these three actors. For this construction is part of a key terminology, the research problem is determined, then the scope with their respective goals are, and then make the methodological development through the application of the axes to the Colombian context; for which a list of the subject of study, definition map with axes and possible futures, then the results are analyzed to generate conclusions and recommendations finally built. The main result of this reflection, evidenced a strong trend in the transformation to a business college, emphasizing training and risk-taking, where some companies operate even as universities.
\end{abstract}

Keywords. Schwartz Shafts, Enterprise, Government, Technology Foresight, Triple Helix, University. 


\section{Introducción}

Este artículo busca construir y aplicar herramientas que ayuden a la validación de metodologías de mejoramiento empresarial, proyección académica y crecimiento gubernamental. Herramientas como la lluvia de ideas y el análisis PESTEL son de gran aplicación en diferentes campos, ayudando a incrementar el valor de los procesos.

En esta oportunidad serán usados para el despliegue del modelo triple hélice, considerando los cuatro ejes que lo componen: a) modelo coopetencia, b) solo pragmatismo, c) innovación cero y d) sin inversión; finalmente será una aplicación la que permita evidenciar su validez y plantear posibles retos futuros sobre la construcción del modelo.

Como parte del problema, se busca dar respuesta a la pregunta de investigación ¿Cómo se pueden evidenciar los alcances de la relación UniversidadEmpresa, en temas de gestión de conocimiento, innovación y desarrollo tecnológico de la sociedad? Este planteamiento busca ser abordado por medio de la metodología de los Ejes de Schwartz.

\section{Terminología y conceptos}

Antes de comenzar con la descripción metodológica, se definen los conceptos de la terminología relacionada con la temática del presente artículo (Ver Tabla 1).

\section{Tabla 1. Terminología}

\begin{tabular}{|c|c|}
\hline Término & Definición \\
\hline Prospectiva & $\begin{array}{l}\text { Concepto introducido por Gaston Berger en } \\
\text { los años 50's como una nueva forma de ver } \\
\text { el futuro. Otros autores importantes son } \\
\text { Michel Godet, Bertrand de Jouvenel y } \\
\text { Pierre Massé en los fines de los años 60's. } \\
\text { De esta se puede decir: } \\
\text { Permite tomar una actitud de contemplación } \\
\text { para ayudar con anticipación a la acción. } \\
\text { Por medio de exámenes de un futuro } \\
\text { "lejano", logra anticiparse a cambios } \\
\text { rápidos. interrogarse sobre las } \\
\text { Permite consecuencias de introducción de nuevas } \\
\text { tecnologías para poder mantener el control. } \\
\text { techente }\end{array}$ \\
\hline $\begin{array}{l}\text { Prospectiva } \\
\text { Tecnológica }\end{array}$ & $\begin{array}{l}\text { Tentativas sistémicas para observar a largo } \\
\text { plazo el futuro de la ciencia, la tecnología, la } \\
\text { economía y la sociedad con el propósito de } \\
\text { identificar las tecnologías emergentes que } \\
\text { probablemente produzcan mayores } \\
\text { beneficios económicos y sociales [1]. }\end{array}$ \\
\hline $\begin{array}{l}\text { Ejes de } \\
\text { Schwartz }\end{array}$ & $\begin{array}{l}\text { Los Ejes de Schwartz permiten construir } \\
\text { escenarios objetivos identificando un foco, } \\
\text { las fuerzas llave y tendencias del entorno, } \\
\text { de esta manera se logra elaborar escenarios } \\
\text { útiles para definir esquemas de decisión } \\
\text { posibles y de contingencia. La metodología } \\
\text { "escenarios" de Peter Schwartz, conduce a } \\
\text { la elección de un "escenario apuesta" que } \\
\text { será el camino a recorrer desde el presente } \\
\text { hacia el futuro [2]. }\end{array}$ \\
\hline $\begin{array}{l}\text { Modelo } \\
\text { Triple Hélice }\end{array}$ & $\begin{array}{l}\text { Es propuesto por Henry Etzkowitz y Loet } \\
\text { Leydesdorff y representa la evolución del }\end{array}$ \\
\hline
\end{tabular}

papel de las universidades en la sociedad basada en el conocimiento, la relación academia, empresa y gobierno. Destacar además las funciones de la universidad empresarial no solo de investigación y de enseñanza, sino también de desarrollo económico por medio del impulso hacia la universidad emprendedora [3].

El tema de estudio central del presente artículo, se basa en la Prospectiva Tecnológica; según, el profesor Carlos Cortes de la Universidad Nacional de Colombia, la prospectiva se puede segmentar de manera vertical y horizontal [4].

\subsection{Segmentación vertical}

- Prospectiva tecnológica

- Prospectiva social

- Prospectiva territorial

- Prospectiva estratégica

\subsection{Niveles (Segmentación horizontal)}

- Global: Organizaciones no gubernamentales. Por ejemplo el cambio climático.

- Sectores económicos: La informática, los automóviles, etc.

- Empresarial: Estrechamente relacionada a las estrategias de la empresa.

Antes de continuar, es importante realizar la salvedad en relación al alcance del presente artículo, a pesar que el Modelo Triple Hélice, contempla las relaciones Universidad-Empresa-Estado, por tratarse de una construcción académica y práctica, solo se realizará desarrollo metodológico en la aplicación de los Ejes de Schwartz sin incluir el tercer componente correspondiente a Estado.

Muchos autores han utilizado estos planteamientos de estrategia a partir de la utilización de prospectiva con el ánimo de generar planes de acción y procesos de toma de decisiones. Uno de esos casos, es el expuesto por Sánchez, Montoya y Montoya en su artículo: Aplicación del enfoque integrado de prospectiva y estrategia para el mejoramiento al proceso de selección docente de la Universidad Nacional de Colombia, en el cual por medio del enfoque integrado de prospectiva y estrategia, concluyen en relación a los cambios institucionales que pueden resultar de la generación de proyectos con base en propósitos de mejoramiento continuo en el área específica de estudio, en este caso, resultando estrategias puestas en marcha desde los diferentes estamentos administrativos [5].

\section{Problema de investigación}

Para el planteamiento del problema se recurre a la siguiente pregunta: ¿Cómo se pueden evidenciar los alcances de la relación Universidad-Empresa, en temas de gestión de conocimiento, innovación y desarrollo tecnológico de la sociedad? Será a través de esta pregunta que se buscará evaluar el paradigma académico empresarial considerando del modelo de los Ejes de Schwartz en temas de prospectiva tecnológica. 
Ingenierías USBMed, Vol. 8, No. 1, Enero-Junio 2017

\section{Alcance}

La construcción de este artículo comienza con la revisión de conceptos referentes al tema de estudio, para luego utilizar la metodología de análisis de los Ejes de Schwartz al tema de prospectiva tecnológica en la relación Universidad-Empresa y finalmente concluir a la luz de los resultados.

Para comprender el desafío que tiene hoy en día la empresa y la universidad, como actores principales del presente escrito, es necesario enfrentarse a un futuro volátil, mejorando las predicciones, estimular la intuición y conducir a una acción eficaz. [6]

\section{Objetivos}

- Evaluar la efectividad de las relaciones UniversidadEmpresa con base en la aplicación del modelo de los Ejes de Schwartz en temas de prospectiva tecnológica.

- Ampliar las relaciones académico-industriales por medio de la interacción, el emprendimiento y las alianzas con el gobierno para la promoción de proyectos y la adquisición de recursos.

- Evidenciar cómo el papel tradicional de la universidad se renueva para ayudar a la modernización de las empresas de baja y media tecnología.

- Potencializar la universidad como un recurso para mejorar los entornos de innovación y crear un régimen de economía basada en el desarrollo de la ciencia.

\section{Desarrollo metodológico}

Para la construcción de la metodología se parten de los pasos propuestos para la generación de prospectiva tecnológica, los cuales parten del listado de tendencias del tema de estudio, luego se diseña y se define el mapa con los Ejes de Schwartz y finalmente se plantean los retos futuros. Estos pasos serán aplicados al tema en estudio con el objeto de simular escenarios futuros en la relación Universidad-Empresa.

\subsection{Lista del tema en estudio}

Por medio de este primer paso se busca generar un listado de posibles tendencias, acontecimientos, variables que puedan afectar o tener incidencia sobre el tema en estudio. Dentro de algunos de los métodos utilizados para llevar a cabo este paso se tienen la lluvia de ideas, el análisis DOFA y el análisis PESTEL. Para tener una amplia perspectiva sobre los escenarios a desarrollar, se utilizarán dos de estos métodos.

\subsubsection{Lluvia de ideas}

Este método busca generar una lista de ideas según un tema particular, en este caso, busca conocer a manera de diagnóstico el estado actual de la universidad y la empresa en relación a la gestión de conocimiento, innovación y desarrollo tecnológico que estos dos actores le aportan a la sociedad. A continuación se listan las principales ideas, que surgen de la experiencia y observación directa de los autores, soportados en informes, noticias y datos reales. a. La universidad posee grupos de investigación en pro de la inventiva y el desarrollo tecnológico. En las diferentes universidades del país se cuenta con grupos de investigación dedicados entre muchos temas al desarrollo tecnológico. Según fuentes de Colciencias, para el año 2015, se tenían registrados 6.768 grupos por el GrupLAC, los cuales participan activamente convocatorias a nivel nacional. 4610 de ellos, fueron avalados y reconocidos como Grupos de Investigación, Desarrollo Tecnológico o de Innovación, clasificados además en diferentes categorías, Grupos A1: 382, Grupos A: 546, Grupos B: 977, Grupos C: 1848, Grupos D: 681. [7]

b. La empresa posee áreas destinadas a la I+D. Un estudio realizado por PricewaterhouseCoopers, validó a través de 505 entrevistas a líderes de la innovación en 467 empresas de todo el mundo, que parte del éxito en las organizaciones está en su capacidad de avanzar en innovación a partir del desarrollo de una serie de estrategias alineadas a las necesidades del cliente, desarrollo y retención de personal técnicos, comprensión de nuevos productos y tecnologías, valores agregados en servicio y tendencias, además de búsqueda y desarrollo de nuevos productos, todo esto dentro de la misma organización y gracias a sus departamentos de I+D. [8]

c. La empresa invierte en sus productos y contrata los servicios de la universidad para la realización de sus pruebas y análisis. Industrias Haceb S.A. es un ejemplo de proyección universidad-empresa. A la fecha, posee dos proyectos de invención en trámite: un quemador con un nuevo calentador en apoyo de la Universidad de Antioquia, y una nueva estufa con eficiente desempeño con apoyo de la UPB. Otro ejemplo es el SENA, con el que la compañía ha realizado 4 convenios de formación especializada, para 2.600 personas, con una inversión cercana a los 700 millones de pesos. [9]

d. Desde las universidades se fomenta el emprendimiento y la gestión de empresas con base tecnológica e inventiva, esto abona participación en ciencia y desarrollo económico para el país. Bolívar (2013) en su artículo de investigación, concluye "Las formas que han tomado las estrategias de formación, entre ellas los semilleros de investigación, son tantas como semilleros hay, pues no es posible siquiera afirmar que hay una sola modalidad de semillero al interior de las instituciones. Lo cierto es que por esas múltiples vías, investigación formativa o no, los semilleros han aportado a la formación de una nueva generación de investigadores que se encuentran articulados a grupos y que producen diariamente nuevo conocimiento "por placer" pero dentro de los cánones de las comunidades académicas." [10]

e. Distorsión del modelo de propiedad intelectual al desarrollar innovación, no hay mucha claridad sobre la atribución y la autoría en la gestión de proyectos. En Colombia actualmente se encuentran falencias en cuanto a las leyes de propiedad intelectual y autoría en 
Ingenierías USBMed, Vol. 8, No. 1, Enero-Junio 2017

gestión de proyectos resultando ser una barrera principal en el desarrollo, creación y proyectos de innovación que involucren temas de propiedad intelectual, por ende se ve afectada la capacidad competitiva y desarrollo económico del país. Por esta razón es imperante según Portafolio (2011), capacitar a entidades, instituciones, entre otras organizaciones en estos temas que repercuten directamente el campo de la investigación y desarrollo como eje principal del país. [11]

f. Creación en algunas empresas de centros de $1+D$, donde se involucra a las universidades para el desarrollo de proyectos, ampliando y fortaleciendo los lazos entre la academia y la industria. Con ayuda de entidades y corporaciones como Ruta N, Corporación Tecnnova y Colciencias se desarrolla conjuntamente actividades de ciencia, tecnología e innovación para la creación de modelos de SPINOFF como centros de creación de I+D para el auge de servicios y productos desde las IES dinamizando la economía en el país. [12]

g. Poco fomento de la innovación haciendo que se genere la "fuga de conocimiento" la cual se da cuando el trabajador logra en sus estudios avances pero no son valorados. Tomando como referencia estudios de emigración calificada, "es importante destacar que el caso colombiano representa un gran reto para los entes gubernamentales, especialmente Colciencias, en tópicos relacionados con la centralización y sistematización oficial de la información sobre las migraciones... El marco institucional de la cooperación internacional en CTel debe trascender de los acuerdos entre países, para materializarse en oportunidades de retorno para el capital humano especializado". [13]

h. Modelos de transferencia tecnológica que incentiva a los programas de emprendimiento regional y nacional. En su artículo de investigación, Taylor (2016), muestra como la cooperación internacional para el desarrollo (CID) juega un papel importante en los Objetivos de Desarrollo Sostenible (ODS) y el modelo propuesto de intraemprendimiento incluyente, por lo que es una herramienta útil para enfrentar la pobreza y cerrar la brecha de la desigualdad, este como ejemplo de los muchos modeles de transferencia orientada a la gestión y economía del conocimiento. [14]

i. Pocas garantías para la gestión de las ideas innovadoras, destinando recursos para el $I+D$ con altos niveles de burocracia. Se evidencia el gran reto de la universidad por generar alianzas y esfuerzos colectivos no solo con la empresa sino también con el estado, debido a la carencia de objetivos comunes, o iniciativas de conveniencia mutua, Orozco (2014) en su editorial plantea una pregunta “¿Están preparadas las universidades para contribuir al desarrollo social, más allá de la función de formación de talento humano?" no por el ejercicio de formación, sino por los muchos impedimentos que empresa y estado pueden generar para su participación activa. [15] j. Poca retroalimentación entre los actores, Empresa/Universidad, sobre los resultados de las investigaciones realizadas en forma conjunta. Según estudios de la OCDE (Organización para la Cooperación y el Desarrollo Económicos), de las políticas de innovación en Colombia, el sistema de innovación es pequeño y no se cuenta con un eje central empresarial como líder. De otro lado, el gasto en I+D es de solo el 0,2\% del PIB siendo mínimo si se compara con países vecinos como Brasil y Chile. Esto ayuda a que los actores Empresa-Universidad tengan diferentes formas de retroalimentación e independencia para con el Estado como eje principal en las relaciones conjuntas. [16]

k. El gobierno nacional, junto con la empresa apoyan de manera significativa la investigación y el desarrollo social de la Universidad. Según la Revista Dinero en una de sus publicaciones, el gobierno nacional planea duplicar la inversión en las actividades de ciencia, tecnología e innovación, reunidas bajo el acrónimo de $\mathrm{ACTI}$, de $0,5 \%$ hoy a $1 \%$ del PIB en 2018. Estos recursos ascenderán a $\$ 8,8$ billones. Esto como aporte significativo al desarrollo social de los diferentes ejes de investigación. [17]

\subsubsection{Análisis PESTEL}

Este método se basa en el entorno externo de la empresa o actor que está siendo estudiado, considera aspectos Políticos, Económicos, Sociales, Tecnológicos, Ecológicos y Legales, que permiten identificar el impacto que éstas puedan llegar a tener de manera interna. A continuación se identifica cada uno de estos aspectos en relación a la gestión de conocimiento, innovación y desarrollo tecnológico que la universidad y la empresa le aportan a la sociedad.

I. Políticos: Nuevas leyes gubernamentales que restringen el uso de los recursos destinados a la investigación tecnológica. Revista de Ciencias Administrativas y Sociales, Innovar, menciona que una de las restricciones políticas está dada por las exigencias de calidad académica, impulsadas por el Ministerio de Educación Nacional MEN, aunque han fomentado obligatoriamente la mejora en la investigación, la falta de recursos económicos, en algunas universidades, restringe la investigación tecnológica necesaria para impactar positivamente el entorno. Agregando "las exigencias de las entidades de dirección y control de la educación superior en Colombia, principalmente lo referente al registro calificado y los procesos de acreditación de alta calidad". [18]

m. Económicos: Dificultad de acceso a recursos para el desarrollo y la gestión de proyectos tecnológicos en la Universidad. Según la Revista de Ciencias Administrativas y Sociales, Innovar, quienes realizaron un artículo, sobre los estímulos y las restricciones en investigación, concluyeron, mediante una encuesta online a 193 investigadores y 63 decanos, que independiente de la cantidad de 
recursos económicos que demande la investigación, al momento de hacer un balance, se puede afirmar que los recursos son una limitante para esta actividad: sólo el $15,1 \%$ de los investigadores encuestados percibe que existen suficientes recursos financieros en el país para ello. [18]

n. Sociales: Generación de impactos en la comunidad por medio del desarrollo de proyectos tecnológicos. A pesar del alto nivel de corrupción que se vive internamente en Colombia, la investigación seria, siempre tiene un impacto positivo en la comunidad. Según un informe sobre el Panorama actual en innovación social, desarrollado por el BID, podría decirse que Medellín, como ciudad, y Antioquia, como departamento en que ella se encuentra, llevan unos 10 años más de experiencia en inversión social, IS que el ámbito nacional. [19]

o. Tecnológicos: Importación de tecnología de punta en el país por medio de empresas líderes en el mercado. Entre las empresas líderes y algunos actores del estado se unen para importar maquinaria desde Milán Italia con tecnología de punta para el sector textil la cual permitirá a los empresarios realizar cortes y terminaciones prefectas de sus prendas. Empresas líderes en el mercado como Vestimundo, Balalaika, Grupo Éxito, grupo Esprit, entre otras cuentan con este tipo de tecnología generando ventaja en energía y ahorro de tiempo. [20]

p. Ecológicos: Normatividad de disminución de impactos negativos a partir de la innovación de tecnología. Entre mejores avances hayan en innovaciones tecnológicas y desarrollos de productos, servicios, procesos se debe de buscar la minimización de impactos ambientales en la utilización de servicios blando y duros. [21]

q. Legales: Disminución de impuestos, partiendo del desarrollo de la tecnología. Según la ley 1450 de 16 de Junio de 2011, en el artículo 158-1, se realiza la reducción por inversiones en investigación y desarrollo tecnológico. [22]

r. Mundiales: Los países vecinos abren las puertas para la investigación y el desarrollo empresarial y académico. Se han encontrado estudios que validan como hasta aproximadamente el año 2010, existían más de treinta asociaciones con integrantes originarios de países de América Latina y el Caribe, divididas en categorías como: asociaciones de estudiantes, gremios y profesionistas; asociaciones locales de profesionistas; asociaciones de empresarios y redes de intelectuales y científicos. Cada de una de ellas con un denominador común: identificarse con un Estado- Nación y contribuir a la agenda de desarrollo de sus países. [14]

\subsection{Definición de mapa con ejes}

Una vez generada la lista de variables, tendencias o acontecimientos en relación al tema en estudio, en este segundo paso, se define el mapa con los Ejes de Schwartz, cuya construcción determinan los cuatro escenarios posibles en donde cada una de las ideas del paso anterior, serán ubicadas dependiendo del enfoque o definición de cada uno de estos escenarios, estos son: Modelo coopetencia, Solo pragmatismo, Innovación cero y Sin inversión [2].

La Figura 1, muestra el resultado del cruce de los ejes, considerando dos de los actores del Modelo Triple Hélice Universidad-Empresa, generando relaciones positivas $(+)$ y negativas $(-)$ entre ellos.

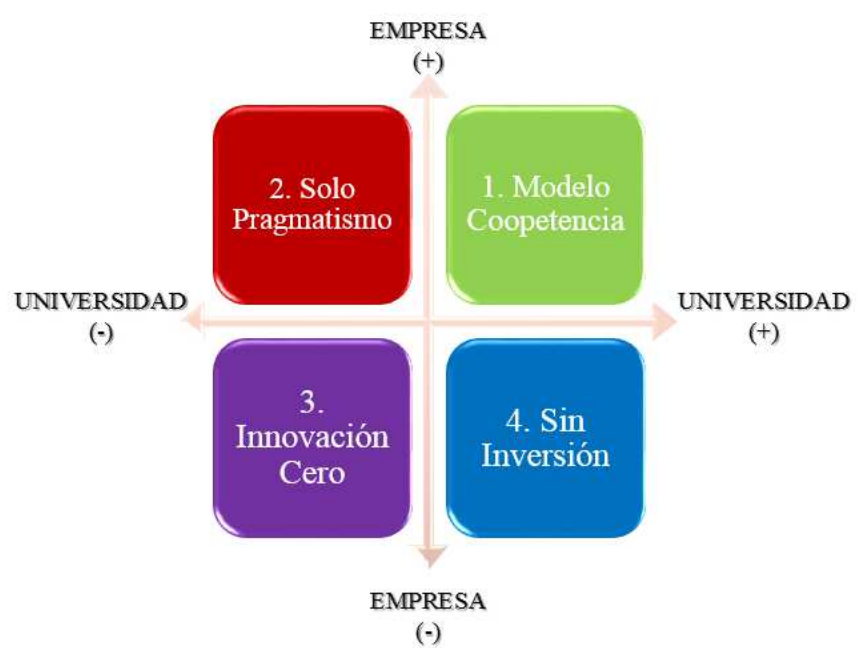

Figura 1. Ejes de Schwartz a la relación UniversidadEstado

A continuación se explica en detalle lo que representa cada uno de estos escenarios y las relaciones existentes entre ellos.

\subsubsection{Modelo coopetencia}

Hace referencia al estado positivo entre ambos actores Universidad-Empresa, lo cual representa una relación gana-gana, existiendo un evidente interés de oferta y demanda por el tema de investigación en tecnología. La coopetencia se presenta a partir de relaciones complementarias, como se da entre los actores Universidad-Empresa, donde la formación de profesionales genera cambios en las empresas constantemente. [23]

\subsubsection{Solo pragmatismo}

En este escenario, la relación es positiva para la empresa, pero negativa para la universidad, en vista que a pesar que genera desarrollo de estrategias y operaciones en los sectores empresariales, no son considerados por ellos los diferentes modelos investigativos y de innovación desarrollados por la universidad.

\subsubsection{Innovación cero}

Para este escenario, los dos actores EmpresaUniversidad, trabajan de manera independiente sin considerar algún tipo de acuerdo, marco, alianza o relación para la gestión de nuevos conocimientos tecnológicos e investigativos. 


\subsubsection{Sin inversión}

En este último escenario, la relación positiva para la universidad, pero no para la empresa. En este la universidad desarrolla nuevos conocimientos partiendo de su estructura investigativa, pero no es aprovechado por la empresa para el desarrollo estratégico y operativo de la empresa.

\subsection{Futuros posibles}

Finalmente, es este último paso, se asocian cada una de las ideas obtenidas en el paso uno de la metodología a los escenarios anteriormente descritos en el paso dos, dando como resultado la Figura 2. Con este se logra generar entonces, dar respuesta en relación a la evidencia de los alcances en la relación UniversidadEmpresa, en temas de gestión de conocimiento, innovación y desarrollo tecnológico de la sociedad a través de los futuros posibles.

\section{Análisis y resultados}

Una vez se realiza la asociación de cada una de las ideas obtenidas en el paso uno de la metodología a los escenarios descritos en el paso dos, se obtienen los siguientes futuros posibles, análisis y resultados.

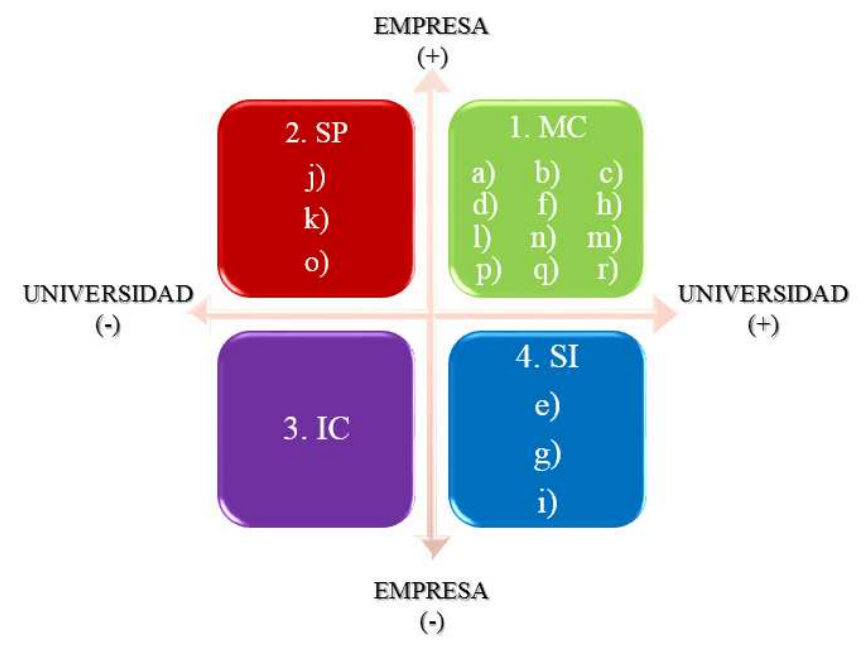

Figura 2. Aplicación de los Ejes de Schwartz a la relación Universidad-Estado

\subsection{Modelo coopetencia}

Se observa una tendencia a mantener la relación gana-gana entre ambos actores, Universidad-Empresa, donde ambos aportan ayudas para la gestión de nuevo conocimiento. Muestra de ellos son los grupos de investigación, áreas de I+D en las empresas, prácticas de pruebas de laboratorios, emprendimiento de base tecnológica, fortalecimiento de lazos académicos e industria, transferencia tecnológica regional y nacional, impacto en la sociedad con proyectos tecnológicos, consideración ambiental desde la investigación, beneficios económicos y legales.

Este escenario representa el $60 \%$ de participación de todo el modelo de los ejes de Schwartz.

\subsection{Solo pragmatismo}

El resultado arrojado representa cómo ante la posible carencia de recursos en investigación tecnológica por parte de la universidad, la empresa buscará la manera de mitigar su problemática con recursos y estamentos legales externos. Muestra de esto son los diferentes convenios marco entre estamentos nacionales e internacionales para la socialización y gestión del conocimiento tecnológico. Este escenario representa el $20 \%$ de participación de todo el modelo de los ejes de Schwartz.

\subsection{Innovación cero}

No es un escenario factible para la proyección de tecnología en el país, debido a que de cierta manera, cualquiera de los dos actores evidencias comportamientos de gestión en tecnología. Este escenario representa el $0 \%$ de participación de todo el modelo de los ejes de Schwartz.

En las Universidades y Empresas, el futuro no es posible sin innovación, el concepto de innovación, es mucho más que una filosofía necesaria en el ámbito empresarial. [24]

\subsection{Sin inversión}

Este escenario final muestra que a pesar de que la universidad genera esfuerzos por incrementar el conocimiento, la innovación y la tecnología, existen riesgos, manejos indebidos y miedos en cuanto a la propiedad intelectual. Muestra de ello es además la deserción de los investigadores de las empresas, y la tramitología para la economía del conocimiento. Este escenario representa el restante $20 \%$ de participación de todo el modelo de los ejes de Schwartz.

\section{Conclusiones y recomendaciones}

Ante la efectividad de la relación UniversidadEmpresa en temas de investigación e innovación tecnológica, se tienen las siguientes conclusiones:

- Se evidencia como las circunstancias locales (independiente si se trata de un país en vía de desarrollo industrial o avanzado), pueden actuar como limitantes sobre la velocidad a la cual las nuevas estructuras institucionales pueden ser constituidas.

- Es clara una fuerte tendencia en la transformación hacia una universidad empresarial, haciendo hincapié en el espíritu empresarial, empresa de la formación y la toma de riesgos, considerando además que algunas empresas también actúan como universidades.

- Se descarta la teoría de algunos observadores que pronostican que la universidad está en el proceso de ser reemplazado por otras formas de organización más especializados, tales como empresas de consultoría, debido al hecho de la necesidad de una sociedad basada en el conocimiento, donde la universidad desarrolla competencias a través de su enseñanza y el aprendizaje en función de la reproducción y la generación de conocimientos. 
- Los ejes de Schwartz como metodología de prospectiva contribuyen a identificar los elementos direccionadores y bajo un enfoque prospectivo de largo plazo considerar los posibles escenarios y niveles de ocurrencia según el entorno en estudio, en este caso la relación Universidad-Empresa.

- A manera de recomendaciones se tiene:

- Es necesario continuar con la invención de nuevas funciones y las relaciones de las instituciones estáticas, promoviendo cambios hacia una mayor dependencia económica en función de la producción de conocimiento.

- Identificar y orientar las tendencias futuras en la producción de conocimiento y sus implicaciones para la sociedad, considerando la asistencia de programas de financiación nacional e internacional para estos desarrollos, con el ánimo de impulsar las actividades de innovación en formatos de la red.

- Se sugiere que los vínculos entre los sectores, que unen las diferentes etapas del proceso de innovación sufran un cambio importante en cuanto al modelo de funcionamiento academia (universidades, laboratorios), empresa (laboratorios de las grandes corporaciones, prácticas empresariales) y gobierno (fuentes de financiación).

- Se deberá continuar con el fomento de nuevos modelos movilidad, tanto de conocimiento y como de los investigadores, de forma tal que se logre articular sistemas de innovación con estructura socioeconómica más "abierta" y menos "restrictiva" en el acceso público a los conocimientos que produce, por ejemplo: promoción de los sistemas de patentes.

- Se hace interesante ampliar esta metodología de prospectiva tecnológica de los ejes de Schwartz a los demás actores que forman el modelo de Cuatro Hélices, es decir, ya se generó un ejercicio sobre la Universidad y el Estado, queda el reto sobre el Estado y la Sociedad.

\section{Referencias}

[1] E. Janstch. "Tecnological Forecasting in perspective". París, OCDE. 1967.

[2] P. Schwartz. "The Art of the Long View: Planning for the Future in an Uncertain World". Nueva York: Bantam. 1991.

[3] R. Zaballa. "El Modelo de la Triple Hélice". Parque de innovación La Salle. Club Asturiano de la Innovación. 2011.

[4] C. Cortes; J. Escamilla. "Gestión Tecnológica: Prospectiva". Universidad Nacional de Colombia, Facultad de Ingeniería. Departamento de Ingeniería de Sistemas e Industrial. S.F.

[5] B. Sánchez; I. Montoya; L. Montoya. "Aplicación del Enfoque Integrado de prospectiva y estrategia para el Mejoramiento al Proceso de Selección Docente de la Universidad Nacional de Colombia". Innovar, 23 (48), pp. 43-54. Consultado el 01 de septiembre de 2016. Disponible http://www.scielo.org.co/scielo.php?script=sci_arttext\&pid=S0121$50512013000200005 \& \operatorname{lng}=$ en\&tlng=es.

[6] Universidad del Valle, «Planificación Estrategica por escenarios. Peter Schwartz,» Cuadernos de Administración, nº 21, pp. 202-225, 1995.

[7] Colciencias. "Reconocimiento y medición de grupos de investigación, desarrollo tecnológico o de innovación". Abril 11 2016. Consultado el 06 de febrero de 2017. Disponible en: http://www.colciencias.gov.co/sites/default/files/convocatoria/listadop reliminar-resultados-conv737-2015-grupos-versionconsulta.pdf.

[8] J. Ramírez. La República. Globoeconomía. "Empresas que más gastan en investigación y desarrollo no son las más innovadoras". Noviembre 07 2014. Consultado el 06 de febrero de 2017. Disponible en: http://www.larepublica.co/empresas-que-m\%C3\%A1s-gastan-en-
investigaci\%C3\%B3n-y-desarrollo-no-son-las-m\%C3\%A1sinnovadoras_189251.

[9] Mineducación. "Beneficios de la alianza Universidad-Empresa-Estado en la UdeA". Abril 05 2010. Consultado el 06 de febrero de 2017. Disponible en: http://www.mineducacion.gov.co/cvn/1665/w3-article221716.html.

[10] R. Bolivar. "Los modos de existencia de la estrategia de semilleros en Colombia como expresiones de la comprensión de la relación entre investigación formativa y la investigación en sentido estricto. Múltiples lecturas, diversas prácticas”. Ágora USB, V.13, №2, Julio-Diciembre. 2013, pp. 433-441. Consultado el 06 de febrero de 2017. Disponible en: http://revistas.usb.edu.co/index.php/Agora/article/view/113/75.

[11] Rodríguez, D. Portafolio. "Hora de fortalecer la Propiedad Intelectual. El sistema requiere blindarse en lo relacionado con el sistema judicial con el compromiso del público en general.”. Diciembre 23 2011. Consultado el 06 de febrero de 2017. Disponible en: http://www.portafolio.co/opinion/redaccion-portafolio/hora-fortalecerpropiedad-intelectual-126900.

[12] Spin-off Colombia. "Invitación a participar Fortalecimiento para entidades vinculadas, constitución y puesta en marcha de Spin-off universitarias". Agosto 19 2016. Consultado el 06 de febrero de 2017. Disponible http://www.utadeo.edu.co/files/collections/documents/field_attached_f ile/terminos_de_invitacion_spinoff_universitarias.pdf?width $=740$ \&height $=780 \&$ inline $=$ true.

[13] G. Angulo. "Cooperación internacional y emigración calificada: diáspora científica colombiana". Revista Internacional de Cooperación y Desarrollo USB, $\mathrm{N}^{\circ} 3$, Año 2016, pp. 99-114. Consultado el 06 de febrero de 2017. Disponible en: http://revistas.usb.edu.co/index.php/Cooperacion/article/view/2544/22 39.

[14] R. Taylor. "Intraemprendimiento incluyente, modelo de sostenibilidad de empleo y cooperación en el Centro de Emprendimiento Pedro Romero". Revista Internacional de Cooperación y Desarrollo USB, $N^{\circ} 3$, Año 2016, pp. 35-55. Consultado el 04 de febrero de 2017. Disponible en: http://revistas.usb.edu.co/index.php/Cooperacion/article/view/2544/22 39.

[15] M. Orozco. "La Integración Universidad-Empresa-Estado-Sociedad como modelo de desarrollo e innovación social". Revista Ciencia Actual USB, Vol. 3, Año 2014, Editorial. Consultado el 04 de febrero de 2017.2 Disponible en: http://revistas.usb.edu.co/index.php/Cienciactual/article/view/2146/18 56.

[16] OCDE. "Estudios de la OCDE de las Políticas de Innovación: Colombia". 2014. Consultado el 06 de febrero de 2017. Disponible en: https://www.oecd.org/sti/inno/colombia-innovation-reviewassessment-and-recommendations-spanish.pdf.

[17] Revista Dinero. "Colombia le apuesta a la ciencia, tecnología e innovación para alcanzar el desarrollo". Pyme. Agosto 192015. Consultado el 03 de febrero de 2017. Disponible en: http://www.dinero.com/edicion-impresa/pymes/articulo/inversionesciencia-tecnologia-innovacion-colombia/212458.

[18] J. Vivares.; L. Gutiérrez; G. Castaño. "Estímulos y restricciones para la investigación en administración en Colombia". INNOVAR. Revista de Ciencias Administrativas y Sociales, Julio-Septiembre 2013, pp. 5-16.

[19] L. Villa; J. Melo. "Panorama actual de la innovación social en Colombia", Banco Internacional de Desarrollo BID, 2015, pp. 84.

[20] D. Rivera; L. Velásquez. "Desde Italia llega moderna maquinaria para tecnificar el sector textil - confección de Antioquia". Inexmoda. Consultado el 03 de febrero de 2017. Disponible en: http://www.inexmoda.org.co/prensa/Desdeitaliallegamodernamaquinar $\mathrm{ia} /$ tabid/5838/Default.aspx.

[21] M. Peñaloza; F. Arévalo; R. Daza. "Impacto de la gestión tecnológica en el medio ambiente". Revista de Ciencias Sociales Vol.15, N², Marcaibo Junio 2009), pp. 306-316. Consultado el 03 de febrero de 2017. Disponible en: http://www.scielo.org.ve/scielo.php?script=sci_arttext\&pid=S131595182009000200010.

[22] Gerence. "Incentivo tributario para quienes invierten en investigación y desarrollo tecnológico". Consultado el 03 de febrero de 2017. Disponible en: http://www.gerencie.com/incentivo-tributario-paraquienes-invierten-en-investigacion-y-desarrollo-tecnologico.html.

[23] Barry Nalebuff, Adam Brandenburger, Coopetencia, Bogota: Norma, 2005. 
Ingenierías USBMed, Vol. 8, No. 1, Enero-Junio 2017

[24] Rodríguez Castellano Arturo, Hoyos Iruarrizaga Jon, Izaguirre Olaizola Julen, M. Azucena Vicente Molina, Investigaciones Europeas de
Dirección y Economía de la Empresa, Volume 17, Issue 1, JanuaryApril 2011, pp. 17-35 